\title{
A Framework for Monitoring and Measuring a Large-Scale Distributed System in Real Time
}

\author{
Lei Zhan*, Tom Z. J. Fu*, Dah Ming Chiu* and Zhibin Lei ${ }^{\dagger}$ \\ *\{z|011, zjfu6, dmchiu\}@ie.cuhk.edu.hk, Department of Information Engineering, \\ The Chinese University of Hong Kong, Hong Kong, China \\ †lei@astri.org, Applied Science Technology Research Institute (ASTRI), Hong Kong, China
}

\begin{abstract}
Increasingly, Internet services are supported by a large-scale and distributed infrastructure on top of the Internet. This includes Content Delivery Networks (CDNs), Peer-to-peer (P2P) networks for content distribution, and more recently Cloud Computing built out of distributed data centers. Applications include Social networks (e.g. Facebook), content distribution and sharing (e.g. YouTube) and various location-based services and applications (e.g. Google Maps). To ensure proper operation and good QoE, these services need centralized monitoring and control of its operation. In this paper, we report our experience in building such a monitoring system. The key components include a reporting mechanism for capturing the system's state, and a visualization library to help administrator to keep track of how well the system is operating. We applied our monitoring system to support a P2P-assisted video streaming network for broadcasting the 2012 London Olympic Games in Hong Kong. We explain how our system keep track (in real time) of key performance numbers interested by the administrators, such as number of concurrent users, the average rate of contribution from different peers, and the QoE observed at different peer nodes, and illustrate various ways such information is visualized.
\end{abstract}

\section{Categories and Subject Descriptors}

C.2.3 [Computer-Communication Networks]: Network Operations-Network Monitoring; D.2.10 [Software Engineering]: Design-Representation

\section{General Terms}

Management, Measurement

\section{Keywords}

Monitoring, Measurement, Visualization, Large-scale, Realtime

Permission to make digital or hard copies of all or part of this work for personal or classroom use is granted without fee provided that copies are not made or distributed for profit or commercial advantage and that copies bear this notice and the full citation on the first page. Copyrights for components of this work owned by others than ACM must be honored. Abstracting with credit is permitted. To copy otherwise, or republish, to post on servers or to redistribute to lists, requires prior specific permission and/or a fee. Request permissions from permissions@acm.org.

HotPlanet'13, August 16, 2013, Hong Kong, China.

Copyright 2013 ACM 978-1-4503-2177-8/13/08 ...\$15.00.

\section{INTRODUCTION}

Increasingly, many practical Internet services are based on a distributed infrastructure, including P2P systems, CDNs, data centers and cloud computing services. In order to provide reliable services and high quality of user experience, it is necessary to build a monitoring system that let administrators monitor and manage the deployed systems. Such a monitoring system must operate in real-time trying to capture relevant information efficiently, and let administrators visualize the system state effectively. For a large-scale service, building such a monitoring system can be quite challenging, because the size of the information that need be captured in real time, and the many dimensions to visualize the system.

We design a general framework for real time monitoring of distributed systems with the following components: (a) end hosts that performance continuous monitoring of local state information and report it regularly; (b) feedback servers that collect reports from end hosts and aggregate the information; (c) a visualization server that analyzes the information for the whole system and display it in various forms for administrators to visualize the system. Our monitoring system is deployed to support a P2P streaming system for live broadcasting the 2012 London Olympic Games through the Internet within Hong Kong area. We show the measurement results of several key metrics of the system during this event. This experience validates the usability and reliability of our monitoring system.

In the rest of the paper, we discuss general considerations for building such a monitoring system in section 2 , followed by the detailed description of our system in section 3 . In section 4 , we describe the case study of using it with the $\mathrm{P} 2 \mathrm{P}$ streaming system and show various measurement results. Finally, we discuss related works in section 5 and conclude our work in section 6 .

\section{DESIGN OBJECTIVES}

In designing the framework for monitoring and measuring the distributed systems in real time, we need to first establish our design objectives.

Accuracy: achieving accurate result is a primary objective. This requires both data completeness and processing effectiveness. If the measured system is of large scale, sampling techniques should be considered to balance between accuracy and scalability.

Real-time: real-time monitoring is of great value for systems that provide consistent and high quality services. It 
could assist system administrators in understanding the status of the measured system and detecting anomalies quickly.

Visualization: a good representation of measurement results should give system administrators a quick understanding of the state and potential problems of the measured system. Meanwhile, visualization techniques help summarizing the measurement results, which make the system-wide information easy for human consumption.

Scalability: the scale of the measured system varies much in size, for example, the peer number of a P2P live streaming system could oscillate from dozens to millions. The framework should be designed to be scalable.

\section{FRAMEWORK DESCRIPTION}

Figure 1 illustrates the basic structure of our framework, which contains four main components: the end host, the Coordinator, the feedback servers and the monitoring platform. Briefly speaking, end hosts of the measured system periodically generate feedback messages and send to the Coordinator. The feedback messages are then forwarded to feedback servers. The monitoring platform communicates with feedback servers periodically to retrieve available aggregated measurement data for analyzing and visualizing in real time.

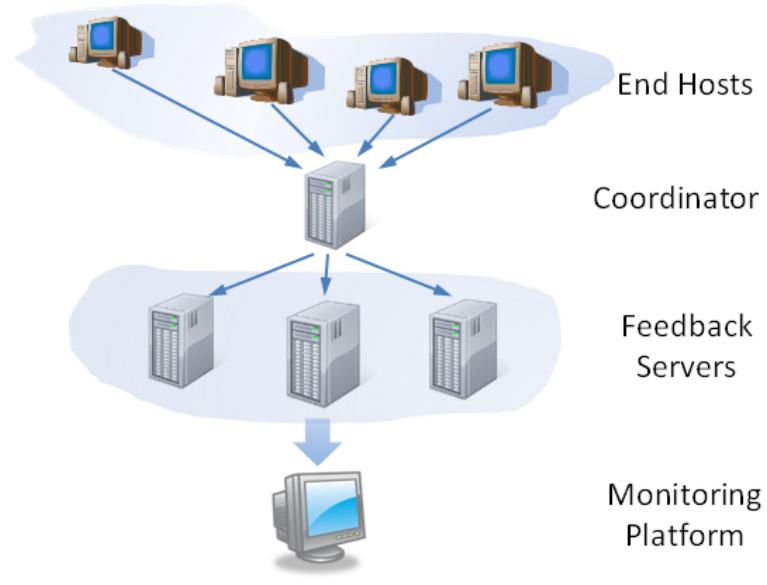

Figure 1: Illustration of basic structure of the framework.

\subsection{End Host}

End host refers to end device or end user of the measured distributed systems, such as a peer in the $\mathrm{P} 2 \mathrm{P}$ network, a data server in the CDN. The end host is the only component of our framework that belongs to the measured system. In fact, the real-time monitoring and measurement is achieved by aggregating and analyzing feedback messages that are generated periodically by a module deployed on end hosts. Even though the functionality is the same, the module should be designed and programmed separately for being compatible with different distributed systems.

Normally, a feedback message generated by the end host contains the unique ID of the generator, the generation timestamp together with some other information of interest. When the messages are centrally processed on the monitoring platform, the unique ID is used to identify the end host, while the timestamp is used to aggregate all feedback messages generated at the same time.

The period for generating and sending feedback message should be configured by system administrators according to the features and actual demands of the measured system. If the measured metric is insensitive with time, it wastes system resources and bandwidth to generate and send feedback messages frequently. Besides, the period configuration should also take the server load of the other framework components into consideration.

\subsection{Coordinator}

The Coordinators located between end hosts and feedback servers (as shown in Figure 1). It is mainly responsible for collecting feedback messages from end hosts and forwarding them to the feedback servers immediately for further processing. It acts as the aggregation point of measurement data, while feedback servers are the backend for recording and processing data.

Certainly, end hosts can directly communicate with the feedback servers, in which case it has to pick a feedback server for connecting whenever it generates a new feedback message. The information of all feedback servers has to be hard-coded into each end host, which leads to problems when modifying the number or the properties of the feedback servers. Therefore, the Coordinator is included in our framework to provide a unique and explicit target for end hosts to send feedback messages. In addition, sampling can also be applied on the feedback messages by the Coordinator if feedback messages present too much load.

The communication between end hosts and the Coordinator is over HTTP so that a) there is no strict limitation on the length of each feedback message and b) the message transmission is reliable. However, the Coordinator may become the bottleneck of the framework since the number of concurrent connections between end hosts and the Coordinator is limited. Multiple Coordinators can be considered to solve such problems.

After receiving a message, the Coordinator immediately picks a feedback server for forwarding according to a certain scheduling rule (e.g. Random, Round-robin) that is preconfigured by the system administrators. Usually, we can locate the Coordinator and the feedback servers at the same place, so that there is no restriction on how they communicate with each other.

\subsection{Feedback Server}

Feedback messages forwarded by the Coordinator are received and written into log files by the feedback server. The common file formats include Binary, CSV, and JSONString, configured by system administrators according to the actual application requirements. For example, a log file in binary saves space, while a log file in JSONString can be processed more efficiently.

The maximum size of a single file is usually limited (it may vary with different file system) and the log file should be of suitable size for easy processing. Therefore, the feedback server should write log files according to a certain rule, which describes the concrete naming algorithm and size restrictions. For example, a log file can be created to record the feedback messages for each single minute.

Besides, the feedback server is also in charge of responding HTTP requests from the monitoring platform. The requests 
include: a) asking for latest available log file name; b) downloading the corresponding data file. What is more, multiple feedback servers can be set for the purpose of load balancing.

\subsection{Monitoring Platform}

The monitoring platform of our framework is mainly responsible for analyzing the measurement data retrieved from the feedback servers and providing different views for visualization by system administrators. It could either retrieve measurement data from the feedback servers periodically for real-time monitoring (real-time mode); or deal with the recorded data files locally for offline analysis (static mode).

In real-time mode, the measurement data should be retrieved in a higher frequency than the frequency in which the end host generates feedback messages. Theoretically, the monitoring platform could run anywhere as long as the Internet is accessible. But the bandwidth between the monitoring platform and feedback servers may be the bottleneck of real-time monitoring. In such case, system administrators could adjust configurations, such as the log file format, to increase the efficiency of data transmission.

Regarding visualization, the monitoring platform provides several views to present the measurement results in different ways. The views should be designed and implemented according to the features of the measured metrics and the interests of system administrators, such as geographic locations, the distribution of some statistics, the change of data over time.

\section{CASE STUDY: 2012 LONDON OLYMPIC GAMES}

To validate the usability and accuracy of our framework, we deployed it in a practical P2P streaming system. The $\mathrm{P} 2 \mathrm{P}$ streaming system is utilized to assist in a live broadcasting event of the 2012 London Olympic Games through the Internet within Hong Kong area.

\subsection{Basic Configurations}

In this case, end hosts correspond to the peers in the measured P2P streaming system. The module for generating and sending feedback messages is taylor programmed and embedded into the video player (a special one designed for this broadcasting event). The period of generating and sending a feedback message is configured to be 30 seconds, in order to avoid overloading the Coordinator. On average, we have $N_{c}=N_{u} / 30$, where $N_{c}$ denotes the number of feedback messages received by the coordinator and $N_{u}$ denotes the number of concurrent users.

\begin{tabular}{|c|c|c|c|c|c|c|c|c|c|c|}
\hline timestam & peerID & \multicolumn{2}{|c|}{ channelID } & \multicolumn{2}{|c|}{ publiclP } & \multicolumn{2}{|c|}{ publicPort } & \multicolumn{2}{|c|}{ privatelP } & privatePort \\
\hline macAddr & \multicolumn{2}{|c|}{ inByteCount } & \multicolumn{2}{|c|}{ outByteCount } & \multicolumn{2}{|c|}{ inBitRate } & \multicolumn{2}{|c|}{ outBitRate } & \multicolumn{2}{|c|}{ numNeighbors } \\
\hline uptime & downloadTin & & lossRat & con & nde & & & QOES & ring & \\
\hline
\end{tabular}

Figure 2: Illustration of data fields in the feedback message.

There are totally 22 fields in a single feedback message and the main fields are listed in Figure 2. The feedback message is constructed as a JSONString and sent to the Coordinator over HTTP. The Coordinator is built on Nginx [1] in version
1.2.2. When forwarding the feedback message, it picks a backend feedback server in Round-robin fashion.

The feedback server runs as a PHP server to respond to requests from the Coordinator. It writes the received feedback message (in JSONString format) directly into plain text log file line by line. Such recording scheme helps reduce the load of feedback server. A new log file is created for every minute to record the feedback messages that are received within a minute. The timestamp in the granularity of minutes is used as the name of the log files. This could simplify the naming procedure and hence the later processing of the measurement data files.

The monitoring platform retrieves log files from the feedback servers periodically, configured to be every 20 seconds.

By sending HTTP requests in each period, the monitoring platform determines and downloads the newest generated $\log$ files from all the feedback servers. In our design, log files on each feedback server are compressed (as a zip file) before transmission. This is to save bandwidth and speed up the transmission.

\subsection{Monitored Metrics}

To obtain and present the measurement results of a particular second, the monitoring platform collects all feedback messages with the timestamp field equal to that second and extracts certain fields to obtain the summarized statistics. A number of key metrics of the $\mathrm{P} 2 \mathrm{P}$ streaming system is measured and monitored in real time. We list these metrics in two categories:

i) System statistics [8]: Number of new peers, Total number of peers, Average peer upload rate, Average peer download rate, Peer contribution ratio;

ii) System performance [6, 8, 9, 14]: Peer Startup Delay, Peer Continuity, Quality of Experience (QoE)

In addition, the monitoring platform provides some filtering functions to flexibly monitor the interested group of peers according to their physical addresses, viewing channels and access ISPs.

\subsection{Visualization Views}

After downloading log files, the monitoring platform processes the files and extracts some statistics for visualization in different views. Figure 3 shows the four views provided by our monitoring platform: a) Map View, b) District View, c) Histogram View and d) Timeline View. Each view focuses on a particular aspect of data or peer properties.

a) Map View: this view utilizes the physical location of peers, which is not contained in the feedback message but determined by IP of the peer. In this view, Hong Kong is divided into 38 areas with approximately equal size and a peer is assigned to an area according to its IP when it joins the network. This view presents the measurement results in spatial domain.

b) District View: this view is similar to the Map View as it also makes use of the physical location of peers. In this view, Hong Kong is divided into 18 areas according to the division of administrative region [2]. This view combines the histogram view together with the map view for explicit observation.

c) Histogram View: this view presents the measurement results in the format of histograms. For a particular 

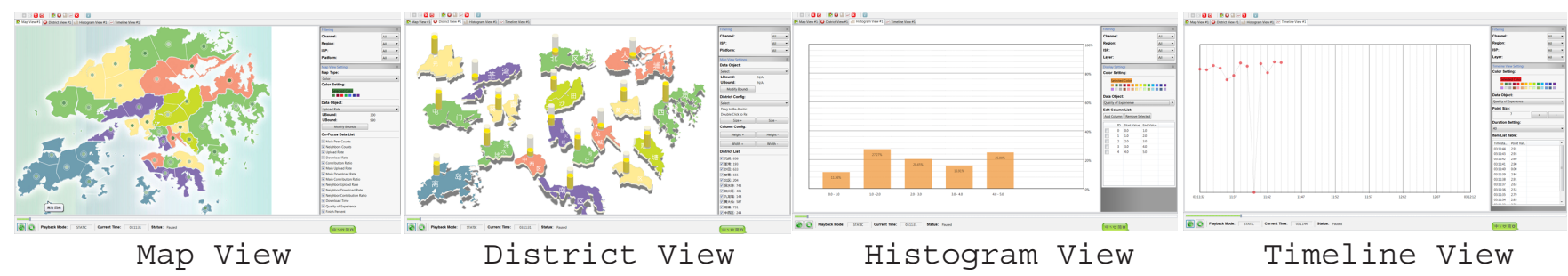

Figure 3: Four views of the monitoring platform (from left to right): Map View, District View, Histogram View and Timeline View.

performance metric, the column represents the percentage of peers that falls into the data range of that column. This view helps system administrators understand the distributions of monitored performance metrics.

d) Timeline View: this view presents the measurement results in time domain. For a particular metric, this view illustrates how the metric value changes over time. By comparing metric values in time dimension, it could help system administrators discover problems or exceptions efficiently.

\subsection{Measurement Results}

The 2012 London Olympic Games lasted for 17 days (July 27 to August 12) and our measurement and monitoring activity covers the whole period. We summarize the complete measurement data and present the changing pattern of some performance metrics along with date in Figure 4.

Figure 4(a) presents the number of newly joined users and total users of each day, together with the cumulative number of distinct users during the whole period. There are a few thousands of new users joining in the system each day and the total number of distinct users reaches 177,245 on the last day.

Figure 4(b) shows the average upload and download rate in kbps of each day. Apparently, these two metrics are consistent along with date and almost all values lie in the range of $800 \mathrm{kbps}-900 \mathrm{kbps}$. Compared to the measurement results of 2008 Beijing Olympic Games, there is a great improvement in network performance as the average upload and download rate of last Olympic Games was only around $500 \mathrm{kbps}$.

Figure 4(c) indicates the average online time per user and per view of each day. Almost all the values of average online time per view are over 40 minutes, which means the video streaming system is providing good services since it attracts reasonably long viewing durations. On average, the user spends more than 1 hour viewing the broadcasting event on the system each day.

Figure 4(d) gives the average continuity index of each day. The values are stable and are above 0.988 during the whole period. Compared to the values measured for the 2008 Olympic Games, the system performed much better in video streaming this time.

Besides the measurement results of the above metrics, Figure $5(\mathrm{a})$ presents the average QoE index values of each day during the whole period and Figure 5(b) shows both the number of concurrent online peers and average $\mathrm{QoE}$ value for every minute on August 07. The calculation of the QoE

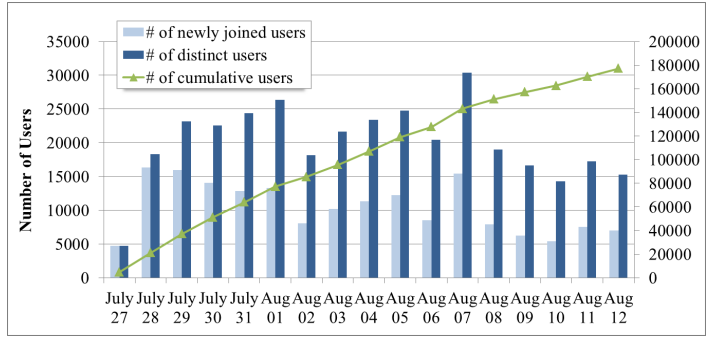

(a)

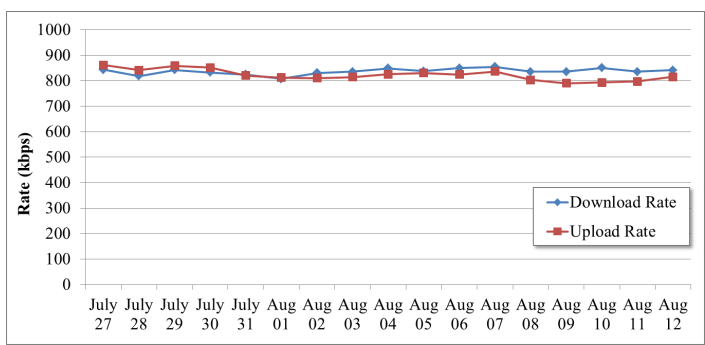

(b)

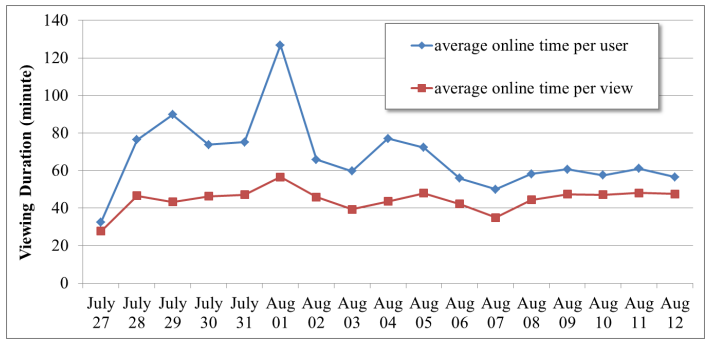

(c)

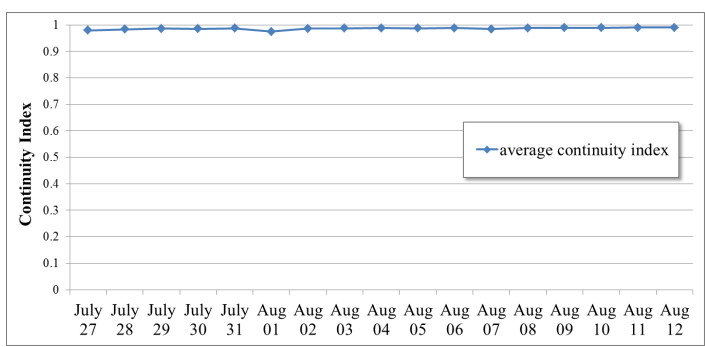

(d)

Figure 4: Demonstration of some metrics of the Olympic Games data.

index is adopted from our previous work [14]:

$$
\operatorname{MOS}(B, D)=c_{1} D+a\left(1-e^{-B \lambda}\right)+c_{2} .
$$




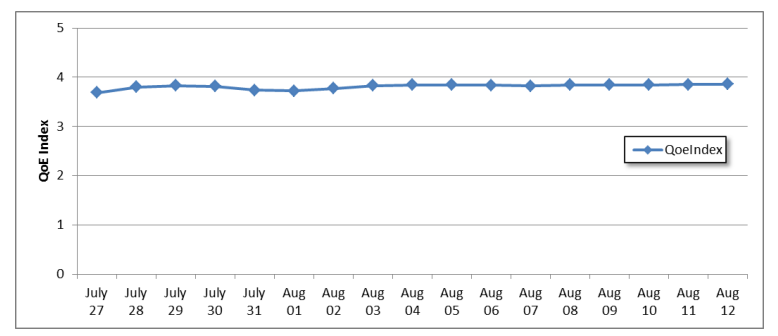

(a)

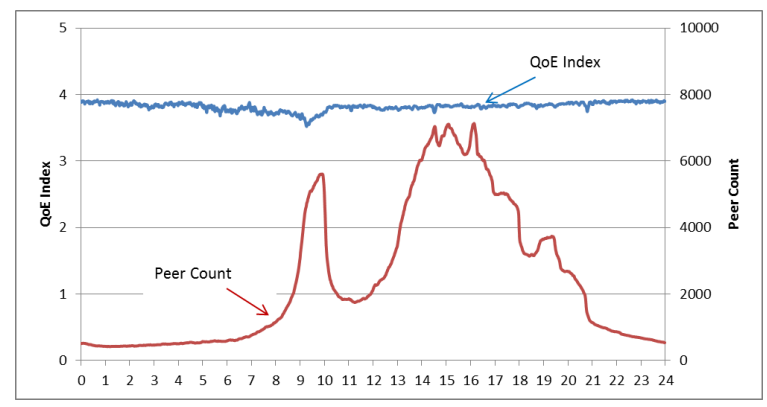

(b)

Figure 5: Demonstration of QoE Index of the Olympic Games data.

In Eq.(1), $B$ stands for video playback rate and $D$ for video discontinuity $\left(=1.0-\right.$ continuity). We set $c_{1}=-5, c_{2}=$ $2, a=4, \lambda=0.0015$, as suggested by [14]. These values are derived by a curve fitting method based on data from subjective testings.

As shown in Figure 5(a), the QoE index values are stable around 3.8 during the whole period of this Olympic Games. (Note, this is calibrated according to a maximum Mean Opinion Score of 5) For a random selected day (Figure 5(a)), we can see more details. One observation is that the QoE value had a temporary dip due to a flash crowd of viewing peers around 9:30am.

In addition, we analyzed the IP coverage of four main ISPs of Hong Kong and the result is listed in Table 1:

Table 1: IP coverage of four main ISPs in HK.

\begin{tabular}{|l|l|l|l|l|l|}
\hline ISP (HK): & PCCW & HKBN & HGC & iCable & Others \\
\hline
\end{tabular}

\begin{tabular}{|c|c|c|c|c|c|}
\hline IP Coverage: & $32.08 \%$ & $13.57 \%$ & $5.55 \%$ & $3.57 \%$ & $45.23 \%$ \\
\hline
\end{tabular}

\subsection{Discussions}

Real-time Delay Analysis: In the case study, the delay of real time monitoring is around 3 minutes. We define delay as the time difference between the generation of the feedback messages and the display of them on the monitoring platform. The total delay is affected by several factors: a) transmission time from end hosts to the Coordinator; b) processing and queuing time at the Coordinator; c) transmission time from the Coordinator to the feedback servers; d) processing and recording time at the feedback server; e) transmission time between the feedback servers and the monitoring platform; f) processing and visualization time at the monitoring platform.

Among all these factors, d) and e) contributed to a large proportion of the total delay, which should be reduced. As for factor d), log files could be created more frequently, such as half a minute instead of one minute in the current setting. As for factor e), we compress the log file before the transmission, which has reduced the size of transmission data to $20 \%$ of the original size. These adjustments could help reduce the delay of real time monitoring.

Feedback Messages Synchronization: In our design, the timestamp of each feedback message is assigned to be the local time at the feedback servers when it is written into the $\log$ file, rather than the time at the client side. This design avoids the time synchronization problem from the client side to the feedback servers. There are only a few feedback servers, so it is possible to synchronize their clocks.

On the other hand, we apply the time window moving average methods to analyze the aggregated feedback messages on the monitoring platform. Such method is widely accepted in real-time systems.

System Scale: According to our measurement results, the total number of distinct users reaches 177,245 and there were thousands of new users joining in the system everyday. The peak value of the number of concurrent online users is around 7,000 and the maximum number of the feedback messages in a single log file is around 13,000. In our measurement, the bottleneck was detected at the monitoring platform as it takes a long time to download the large log files from the feedback servers. It is necessary to redesign the communication pattern between the feedback serves and the monitoring platform to avoid such bottleneck.

Future Works: As we described, the monitoring platform provides Map View and District View, which require us to determine the physical address of peers according to their IP. These views are not used in real-time monitoring for this Olympic Games as the IP geo-location work is not ready. Actually, there exist some methods to do the IP geolocation, for example, we can utilize some public APIs [3]. The integration of the IP geo-location with the monitoring platform in our framework is a direction for future works. In addition, we embed the function of doing traceroute (from an end host to a Google server) into the module that is responsible for generating feedback messages. Therefore, the traceroute records from all active IPs in our system to the Google Server are collected, which will be used in future works, for network tomography. Another important direction is to generalize the framework for monitoring and measuring large-scale and distributed systems, such as distributed data centers.

\section{RELATED WORKS}

Traffic measurement and monitoring for $\mathrm{P} 2 \mathrm{P}$ applications is a well-developed topic, including the measurement studies on P2P file sharing systems [10] and live streaming systems [8]; and the various tools developed for classification and identification on P2P traffics $[7,13]$.

Graph visualization tools, on the other hand, were mostly developed for social network analysis $[4,5]$, such as the Internet graph topology and friendship of human beings, but, in a static form.

There are several prototypes of the monitoring platforms which can visualize the $\mathrm{P} 2 \mathrm{P}$ applications in a dynamic and real-time base, including GnutellaVision [15], VisPastry [12] and OvlVis [11]. However, GnutellaVision [15] and VisPastry [12] are designed for the specific protocols like Gnutella and Pastry, not applicable for other well-known P2P sys- 
tems. OvlVis [11] is designed for generalized purpose use, but not scalable for a large peer population.

\section{CONCLUSION}

We have developed a framework for real-time monitoring and measurement of a large-scale distributed system. The system is general purpose and can be used for $\mathrm{P} 2 \mathrm{P}$ applications, CDN, data centers and cloud computing services, etc. Under the framework, we have implemented a platform to provide multiple metrics visualization for system administrators to monitor and manage the system in real time. The framework is deployed into a commercial P2P streaming system for live broadcasting the 2012 London Olympic Games through the Internet within Hong Kong area. We show the measurement results of several key metrics of the system during this event. The experience shows that such framework can fulfill several hard requirements and is of great value.

\section{REFERENCES}

[1] NGINX, "http://wiki.nginx.org/Main".

[2] The Distric Council of Hong Kong SAR, "http://www.districtcouncils.gov.hk/".

[3] HTML5 Geolocation, "http://www.w3schools.com/html5/html5_geolocation.asp".

[4] Graphviz - graph visualization software, "http://www.graphviz.org/".

[5] K. C. Claffy. Caida: Visualizing the internet. Internet Computing Online, 2001.

[6] T. Z. J. Fu, D. M. Chiu, and Z. Lei. Designing qoe experiments to evaluate peer-to-peer streaming applications. In Visual Communications and Image Processing 2010, pages 77440U-77440U, 2010.

[7] T. Z. J. Fu, Y. Hu, X. G. Shi, D. M. Chiu, and C. S. Lui. Pbs: Periodic behavioral spectrum of $\mathrm{p} 2 \mathrm{p}$ applications. In Proceedings of Passive and Active Measurement Conference (PAM), April 2009.
[8] X. Hei, C. Liang, J. Liang, Y. Liu, and K. W. Ross. Insights into pplive: A measurement study of a large-scale p2p iptv system. In Proceedings of Workshop on Internet Protocol TV (IPTV) services over Would Wide Web, 2006.

[9] Y. Huang, T. Z. J. Fu, D. M. Chiu, J. C. S. Lui, and C. Huang. Challenges, design and analysis of a large-scale p2p vod system. In Proceedings of $A C M$ Sigcomm, 2008.

[10] M. Izal, G. Uroy-Keller, E. Biersack, P. A. Felber, A. A. Hamra, and L. Garces-Erice. Dissecting bittorrent: Five months in torrent's lifetime. In Proceedings of Passive and Active Measurement Conference (PAM), April 2004.

[11] K. Jünemann and J. Dinger. Ovlvis: visualization of peer-to-peer networks in simulation and testbed environments. In Proceedings of the 11th ACM communications and networking simulation symposium (CNS), 2008.

[12] A. Rowstron and P. Druschel. Pastry: Scalable, disributed object location and routing for large-scale peer-to-peer systems. In Proceedings of IFIP/ACM International Conference on Distributed Systems Platforms, 2001.

[13] K. Thomas, P. Konstantina, and F. Michalis. Blinc: Multilevel traffic classification in the dark. In Proc. SIGCOMM '05, 2005.

[14] J. Wang, T. Z. J. FU, D. M. Chiu, and Z. Lei. Perceptual quality assessment on bd tradeoff of $\mathrm{p} 2 \mathrm{p}$ assisted layered video streaming. In IEEE Visual Communications and Image Processing (VCIP), pages $1-4,2011$.

[15] K. Yee, D. Disher, R. Dhamija, and M. Hearst. Animated exploration of dynamic graphs with radial layout. In Proceedings of IEEE Symposium on Information Visualization (INFOVIS), 2001. 